\title{
Determination of Related Substances in Cabazitaxel Using Reverse Phase-liquid Chromatography Method
}

\author{
BAVIREDDI MOHAN', R. S. K. SHARMA', S.V. MURALI MOHANRAO*1 and N.V.S.VENUGOPAL*1 \\ 'Department of Chemistry, GITAM University, Gandhi Nagar, Rushikonda, Visakhapatnam-530045, \\ Andhra Pradesh, India. \\ *Corresponding author E-mail: bavirddim5@gmail.com \\ http/:dx.doi.org/10.13005/ojc/330633
}

(Received: August 09, 2017; Accepted: September 17, 2017)

\begin{abstract}
The present paper describes the reverse phase- high performance liquid chromatographic method and was validated as per $\mathrm{ICH}$ guidelines for the determination of related substances in Cabazitaxel. RP-Liquid chromatography technique was performed with pH 3.0 phosphate buffer and acetonitrile as mobile phase at a flow rate of $0.8 \mathrm{~mL} / \mathrm{min}$. on Waters 2489 UV 2695 pump, Waters 2998 PDA 2695 pump Software Empower ${ }^{2}$ photodiode array detector using Zorbax SB C18 column with UV detection at $220 \mathrm{~nm}$. Linearity was observed in the concentration range of Cabazitaxel LOQ-0.10\% $\left(R^{2}=0.9998\right)$, the concentration range of CBZM01 impurity $0.03-0.225 \%$ $\left(R^{2}=0.9997\right)$, the concentration range of CBZM02 impurity $0.03-0.225 \%\left(R^{2}=0.9997\right)$, the concentration range of CBZN09 impurity $0.03-0.225 \%\left(R^{2}=0.9998\right)$. Limit of detection $(\%)$ and the limit of quantitation $(\mathrm{ng} / \mathrm{mL}$ ) were found to be CBZM01 impurity $0.002 \%$ and $73 \mathrm{ng} / \mathrm{mL}, \mathrm{CBZM02}$ impurity $0.002 \%$ and $71 \mathrm{ng} / \mathrm{mL}$, CBZN09 impurity $0.002 \%$ and $6 \mathrm{ng} / \mathrm{mL}$ and Cabazitaxel $0.002 \%$ and $0.008 \%$ respectively. The percent recovery was in good agreement with the labeled amount in the dosage forms and hence, the method is specific, simple, reproducible and accurate for the determination of Cabazitaxel.
\end{abstract}

Keywords: Cabazitaxel, Estimation of related substances, Liquid chromatography, Percent recovery and dosage forms.

\section{INTRODUCTION}

Cabazitaxel(CBT)(Figure 1.1) was a taxane group chemotherapy drug which is used to treat advanced hormone-refractory prostate cancer'. The IUPAC name of Cabazitaxel was
1S,2S,3R,4S,7R,9S, 10S, 12R, 15S)-4-(Acetyloxy)$15-\{[(2 R, 3 S)-3-\{[($ tert-butoxy) carbonyl]amino\}-2hydroxy-3-phenylpropanoyl]oxy\}-1-hydroxy-9,12dimethoxy-10,14,17,17-tetramethyl-11-oxo-6oxatetracyclo[11.3.1.03,10.04,7]heptadec-13-en-2yl benzoate. CBZ was approved by $\mathrm{EMA}^{2}$ and 
USFDA $^{3}$ for the treatment metastatic prostate cancer $4-5$ in combination with prednisone. Existing bibliographic survey reveals that very few analytical methods have been reported for the determination of Cabazitaxel using Spectrophotometry ${ }^{6}, \mathrm{HPLC}^{7-}$ ${ }^{10}$, and LC-MS/MS ${ }^{11-13}$. Cabazitaxel is not official in any pharmacopoeia and there is no monograph containing methods to determine the Cabazitaxel. Literature survey gives the information on CBZ that there is not even a single method was reported for the determination of related substances in CBZ. Hence, the author was aimed to develop simple, fast and cost effective reverse phase-liquid chromatography method for the determination of related substances in Cabazitaxel and was validated. Chemical structures of related substances of Cabazitaxel such as CBZM01, CBZM02 and CBZM09 were presented in the Figure. 1.2-1.4.

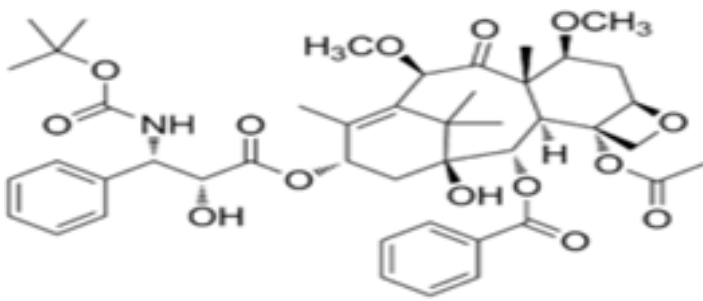

Fig. 1.1 Chemical Structure of Cabazitaxel (CBT)

\section{Chemical structures of related substances:}

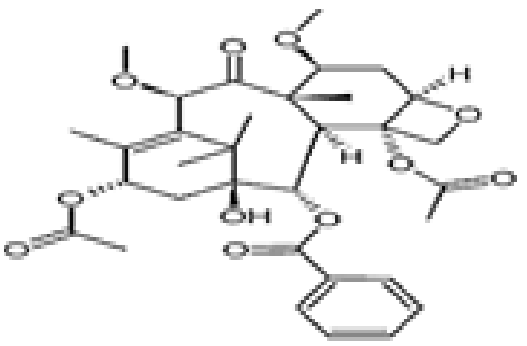

Fig. 1.2 Chemical Structure of 7, 10-dimethoxyDAB (CBZM01)

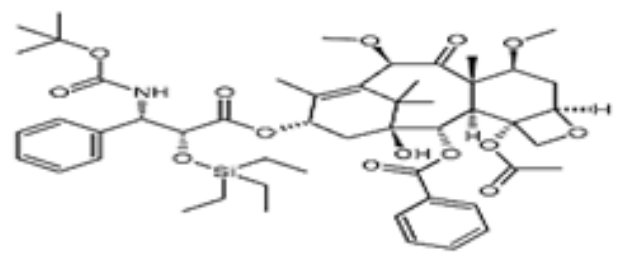

Fig. 1.3 Chemical Structure of TES-Cabazitaxel (CBZM02)

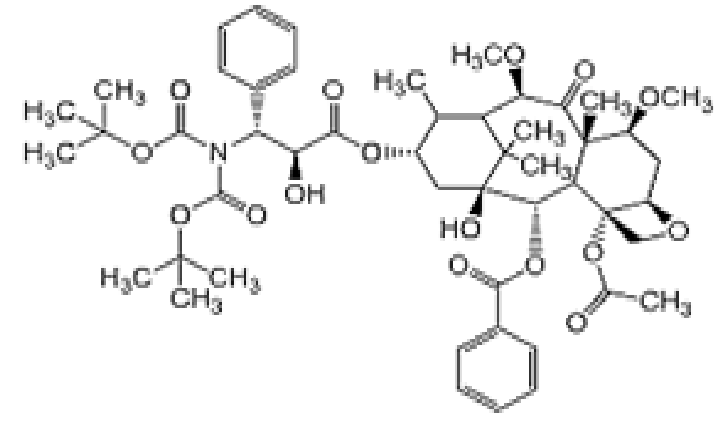

Fig 1.4 Chemical Structure of tigloyl-cabazitaxel (CBZN09),

EXPERIMENTAL

Instrumentation and chromatographic conditions Chromatographic separation was achieved by using a Waters 2489 UV 2695 pump, Waters 2998 PDA 2695 pump Software Empower² photodiode array detector using Zorbax SB C18 $(100 \mathrm{~mm} \times 3.0 \mathrm{~mm}, 1.8 \mu \mathrm{m}$ particle size) column with eluent-A: phosphate buffer eluent-B: acetonitrile as mobile phase at a flow rate of $0.8 \mathrm{~mL} / \mathrm{min}$. with UV detection at $220 \mathrm{~nm}$. Column maintained at temperature $40^{\circ} \mathrm{C}$, sample temperature $10^{\circ} \mathrm{C}$. The overall run time was $22 \mathrm{~min}$. and the flow rate was $0.8 \mathrm{~mL} / \mathrm{min}$. $3 \mu \mathrm{L}$ of sample was injected into the HPLC system.

\section{Chemicals used}

Orthophosphoric acid, Acetonitrile HPLC grade and water were obtained from Merck, India. All chemicals were of an analytical grade and used as received.

\section{Preparation of mobile phase-A}

$1 \mathrm{~mL}$ of $\mathrm{H}_{3} \mathrm{PO}_{4}$ is added to $1000 \mathrm{~mL}$ of water and the solution is properly mixed. Filtered through $0.45 \mu$ membrane filter paper and degassed.

\section{Preparation of mobile phase-B}

Acetonitrile used as mobile phase-B.

\section{Preparation of Diluent:}

$600 \mathrm{~mL}$ Acetonitrile and $400 \mathrm{~mL}$ water are mixed to prepare one litre of diluents and the solution is properly mixed. 


\section{METHOD VALIDATION}

\section{Specificity}

Solutions of CBZM01 impurity, CBZM02 impurity, CBZN09 impurity, and Cabazitaxel each individually prepared and analysed. A spiked solution of each potential impurity to the Cabazitaxel drug substance and analyzed. Analysis was performed by PDA detector and peak purity was determined. Specificity chromatograms of

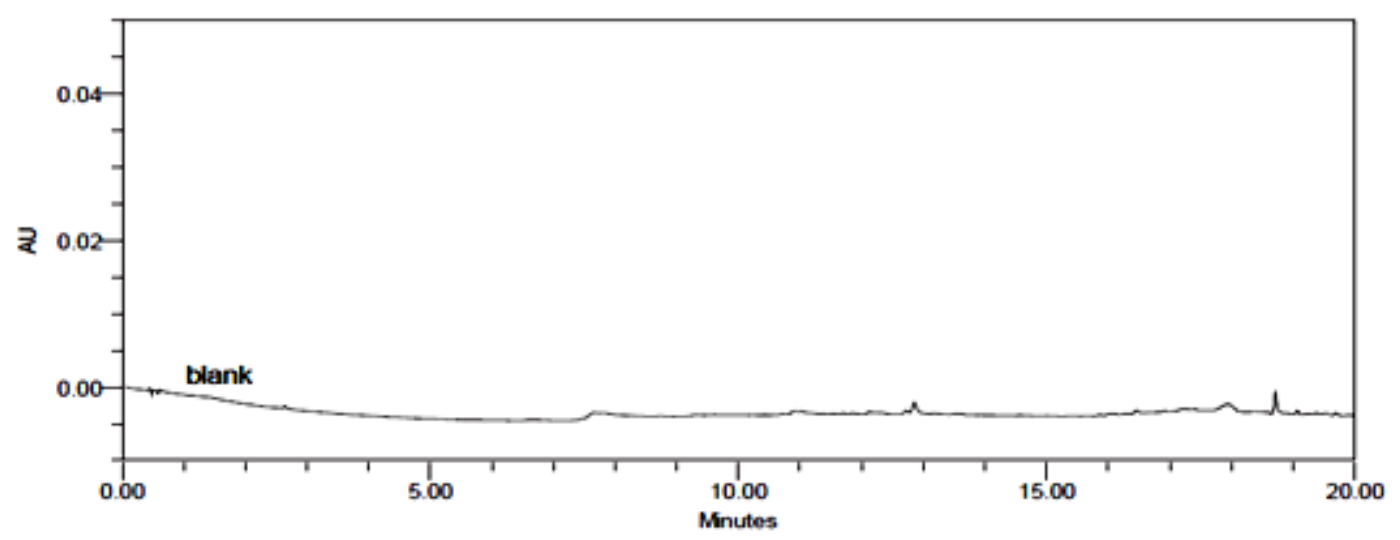

Fig. 1.5: Specificity chromatogram of blank solution

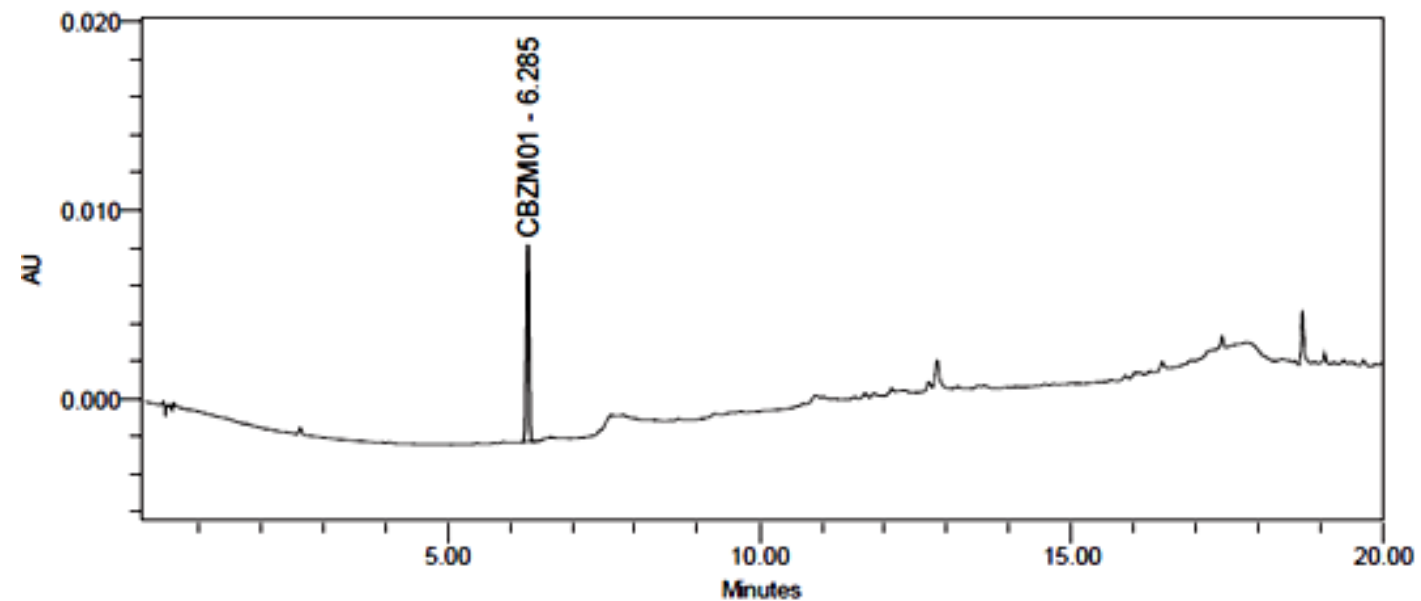

Fig. 1.6: Specificity chromatogram of CBZM01 solution

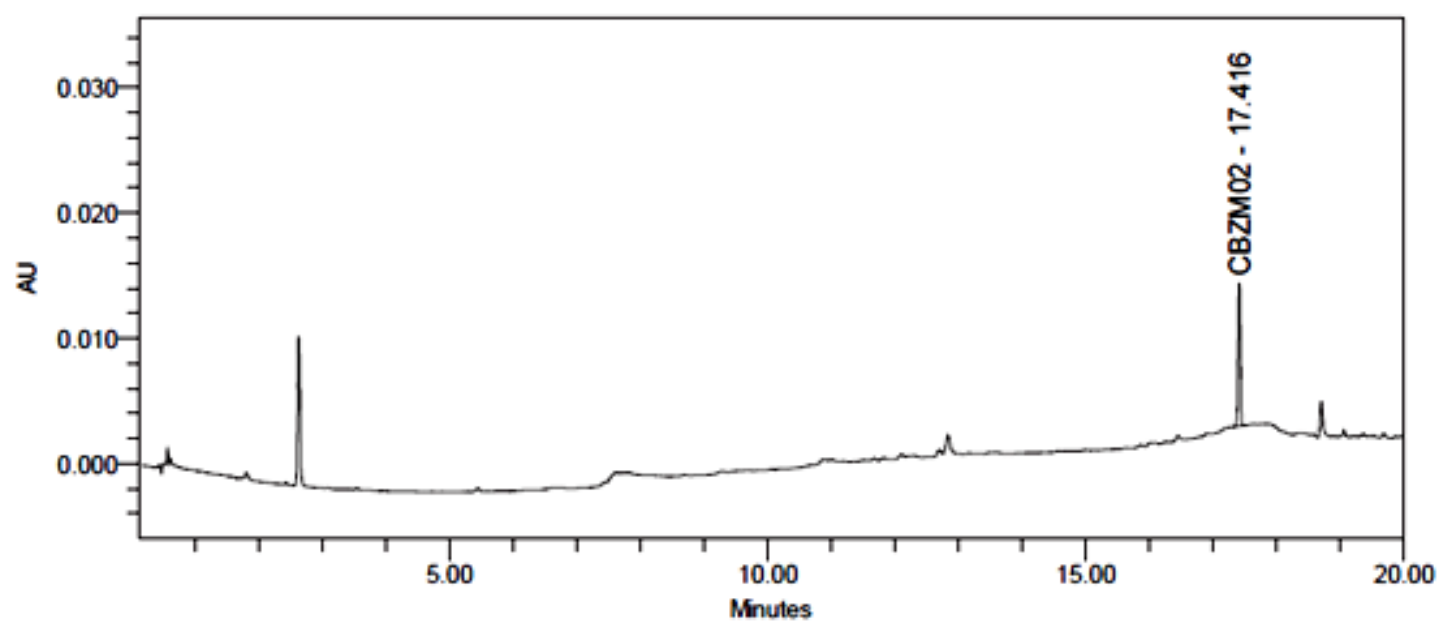

Fig. 1.7: Specificity chromatogram of CBZMO2 solution 
blank, CBZM01,CBZM02,CBZM09 and spiked solution were shown in Figure 1.5-1.9.
The above study reveals that all the known impurities of Cabazitaxel are adequately

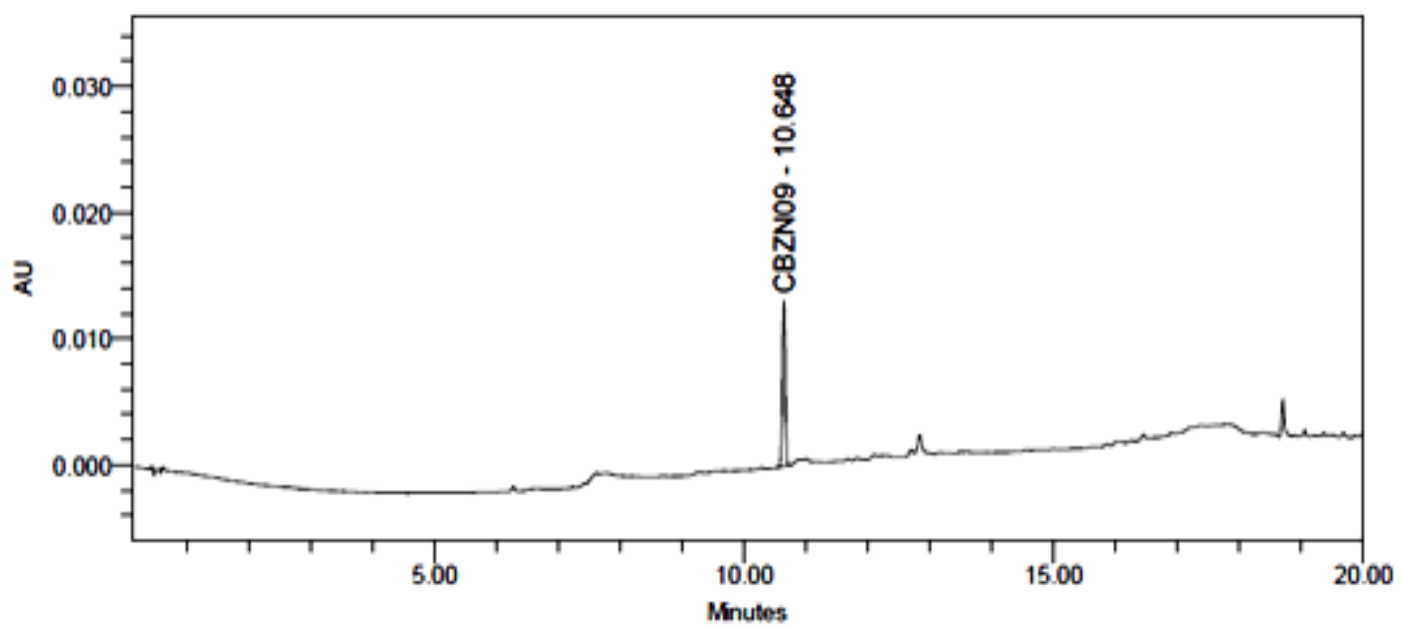

Fig. 1.8: Specificity chromatogram of CBZN09 solution

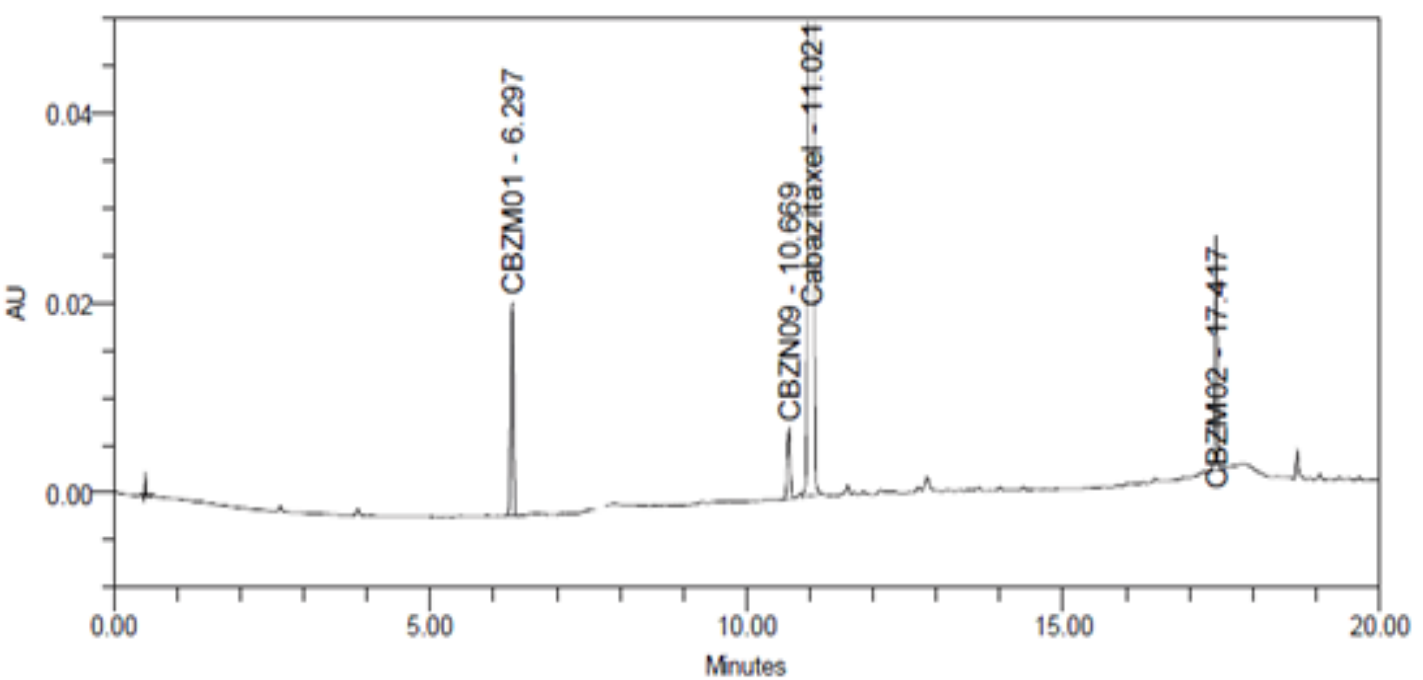

Fig. 1.9: Specificity chromatogram of spiked solution

Table 1.1: Summary of retention time, and relative retention time for known impurities

\begin{tabular}{lcc}
\hline Peak Name & Retention Time & Relative retention time(RRT) \\
\hline CBZM01 & 6.297 & 0.55 \\
CBZM02 & 17.417 & 1.62 \\
CBZN09 & 10.669 & 0.97 \\
Cabazitaxel & 11.021 & 1.00 \\
\hline
\end{tabular}


resolved. Hence, the method is selective for the determination of related substances in Cabazitaxel. Retention time and relative retention time for known impurities were listed in the Table 1.1.

Table. 1.2: Summary of system suitability from standard solution

\begin{tabular}{lccc}
\hline \multicolumn{4}{c}{ Retention times (min) } \\
\hline CBZ & CBZM01 & CBZM02 & CBZN09 \\
10.95 & 6.27 & 17.38 & 10.6 \\
10.99 & 6.29 & 17.37 & 10.64 \\
10.98 & 6.28 & 17.35 & 10.63 \\
10.97 & 6.27 & 17.34 & 10.62 \\
10.95 & 6.23 & 17.34 & 10.6 \\
10.93 & 6.22 & 17.33 & 10.58 \\
10.96 & 6.3 & 17.4 & 10.6 \\
0.2 & 0.4 & 0.1 & 0.2 \\
\hline
\end{tabular}

Table. 1.3: Summary of system suitability from standard solution

\begin{tabular}{lccc}
\hline Sample & CBZ (\%area) & CBZ (area) & $\mathrm{RT}(\mathrm{min})$ \\
\hline Injection 1 & 99.609 & 3929399 & 10.81 \\
Injection 2 & 99.611 & 3929019 & 10.85 \\
Injection 3 & 99.567 & 3936249 & 10.84 \\
Injection 4 & 99.612 & 3932766 & 10.82 \\
Injection 5 & 99.603 & 3931681 & 10.85 \\
Injection 6 & 99.609 & 3932064 & 10.86 \\
Mean & 99.66 & 3931863 & 10.8 \\
RSD (\%) & $<0.01$ & 0.1 & 0.2 \\
\hline
\end{tabular}

\section{System precision}

System suitability was performed by analyzing the reference solution six times. \%RSD for replicate injections of each component from reference solution was calculated. Preparations of Cabazitaxel, CBZM01, CBZM02 and CBZN09 at concentrations of $0.15 \%$ of the nominal concentration of sample required by the method were analyzed in triplicate for each solution. Results of system suitability were presented in the Table 1.2-1.3.

\section{Limit of Detection (LOD) Detection limit of Cabazitaxel}

Stock solution of CBZ standard at concentration level $1.0 \mathrm{mg} / \mathrm{mL}$ was prepared by weighing accurately about $25.029 \mathrm{mg}$ of CBZ standard and was transferred into a $25 \mathrm{~mL}$ volumetric flask, dissolved in diluent and filled up with diluent to the volumetric mark (Table 1.4.). The stock solution was diluted 100times by dispensing $250 \mu \mathrm{L}$ into a $25 \mathrm{~mL}$ volumetric flask, completed with diluent. This $\mathrm{CBZ}$ solution was further diluted into a $25 \mathrm{~mL}$ volumetric flask, dosing $50 \mu \mathrm{L}$. The final concentration (\% level) of CBZ was (0.002 \%). Details of CBZ detection limit was incorporated in the Table 1.5.

\section{Detection limits of the specified impurities}

The stock solutions of individual specified impurities at concentration level $1.0 \mathrm{mg} / \mathrm{mL}$ were prepared $(10.000 \mathrm{mg}$ of specified impurity standard was weighed into a $10 \mathrm{~mL}$ volumetric flask,

Table. 1.4: Stock solution preparation

\begin{tabular}{lcccc}
\hline & Sample Weight $(\mathrm{mg})$ & Dilution $(\mathrm{mL})$ & Potency $(\%)$ & Concentration $(\mu \mathrm{g} / \mathrm{mL})$ \\
\hline CBZ stock & 25.029 & 25 & 0.932 & 933.081 \\
\hline
\end{tabular}

Table. 1.5: Evaluation of $\mathrm{CBZ}$ detection limit

\begin{tabular}{lcccccccc}
\hline S.No & $\begin{array}{c}\text { Sample } \\
\text { weight } \\
(\mathrm{mg})\end{array}$ & $\begin{array}{c}\text { Make up } \\
\text { Volume } \\
(\mathrm{mL})\end{array}$ & $\begin{array}{c}\text { Dilution } \\
(\mu \mathrm{L})\end{array}$ & $\begin{array}{c}\text { Make up } \\
\text { Volume }\end{array}$ & $\begin{array}{c}\text { Dilution } \\
(\mu \mathrm{L})\end{array}$ & $\begin{array}{c}\text { Make up } \\
\text { Volume } \\
(\mathrm{mL})\end{array}$ & Concentration $(\%)$ & $\mathrm{s} / \mathrm{n}$ \\
\hline 1 & 25.029 & 25 & 250 & 25 & 50 & 25 & 0.002 & 3.4 \\
2 & 25.029 & 25 & 250 & 25 & 50 & 25 & 0.002 & 3.9 \\
3 & 25.029 & 25 & 250 & 25 & 50 & 25 & 0.002 & 4.3 \\
Mean & & 3.9 & & & & & \\
\hline
\end{tabular}


Table. 1.6: Stock solutions preparation

\begin{tabular}{lccc}
\hline & Sample Weight $(\mathrm{mg})$ & Dilution $(\mathrm{mL})$ & Concentration $(\mathrm{mg} / \mathrm{mL})$ \\
\hline & 9.301 & 10 & 0.9301 \\
CBZM01_stock & 9.399 & 10 & 0.9399 \\
CBZN09_stock & 9.370 & 10 & 0.9370 \\
\hline
\end{tabular}

Table. 1.7: Evaluation of CBZM01 detection limit

\begin{tabular}{lcccccccc}
\hline S.No & $\begin{array}{c}\text { Sample } \\
\text { weight } \\
(\mathrm{mg})\end{array}$ & $\begin{array}{c}\text { Make up } \\
\text { Volume } \\
(\mathrm{mL})\end{array}$ & $\begin{array}{c}\text { Dilution } \\
(\mu \mathrm{L})\end{array}$ & $\begin{array}{c}\text { Make up } \\
\text { Volume }\end{array}$ & $\begin{array}{c}\text { Dilution } \\
(\mu \mathrm{L})\end{array}$ & $\begin{array}{c}\text { Make up } \\
\text { Volume } \\
(\mathrm{mL})\end{array}$ & Concentration $(\%)$ & $\mathrm{s} / \mathrm{n}$ \\
\hline 1 & 9.301 & 10 & 250 & 25 & 50 & 25 & 0.002 & 3.6 \\
2 & 9.301 & 10 & 250 & 25 & 50 & 25 & 0.002 & 3.4 \\
3 & 9.301 & 10 & 250 & 25 & 50 & 25 & 0.002 & 4.3 \\
\multicolumn{7}{c}{ Mean } \\
\hline
\end{tabular}

.Table. 1.8: Evaluation of CBZM02 detection limit

\begin{tabular}{lcccccccr}
\hline S.No & $\begin{array}{c}\text { Sample } \\
\text { weight } \\
(\mathrm{mg})\end{array}$ & $\begin{array}{c}\text { Make up } \\
\text { Volume } \\
(\mathrm{mL})\end{array}$ & $\begin{array}{c}\text { Dilution } \\
(\mu \mathrm{L})\end{array}$ & $\begin{array}{c}\text { Make up } \\
\text { Volume } \\
(\mathrm{mL})\end{array}$ & $\begin{array}{c}\text { Dilution } \\
(\mu \mathrm{L})\end{array}$ & $\begin{array}{c}\text { Make up } \\
\text { Volume } \\
(\mathrm{mL})\end{array}$ & Concentration $(\%)$ & $\mathrm{s} / \mathrm{n}$ \\
\hline 1 & 9.3700 & 10 & 250 & 25 & 50 & 25 & 0.002 & 3.4 \\
2 & 9.3700 & 10 & 250 & 25 & 50 & 25 & 0.002 & 3.4 \\
3 & 9.3700 & 10 & 250 & 25 & 50 & 25 & 0.002 & 3.2 \\
& & & & & & & & 3.4 \\
\hline
\end{tabular}

Table. 1.9: Evaluation of CBZN09 detection limit

\begin{tabular}{lcccccccc}
\hline S.No & $\begin{array}{c}\text { Sample } \\
\text { weight } \\
(\mathrm{mg})\end{array}$ & $\begin{array}{c}\text { Make up } \\
\text { Volume } \\
(\mathrm{mL})\end{array}$ & $\begin{array}{c}\text { Dilution } \\
(\mu \mathrm{L})\end{array}$ & $\begin{array}{c}\text { Make up } \\
\text { Volume } \\
(\mathrm{mL})\end{array}$ & $\begin{array}{c}\text { Dilution } \\
(\mu \mathrm{L})\end{array}$ & $\begin{array}{c}\text { Make up } \\
\text { Volume } \\
(\mathrm{mL})\end{array}$ & Concentration (\%) $\mathrm{s} n$ \\
\hline 1 & 9.399 & 10 & 250 & 25 & 50 & 25 & 0.002 & 4.3 \\
2 & 9.399 & 10 & 250 & 25 & 50 & 25 & 0.002 & 3.9 \\
3 & 9.399 & 10 & 250 & 25 & 50 & 25 & 0.002 & 4.3 \\
\multicolumn{7}{c}{ Mean } \\
\hline
\end{tabular}


dissolved in diluent and filled up with diluent to the volumetric mark). (Table 1.6). The stock solution was diluted 100times by dispensing $250 \mu \mathrm{L}$ into a $25 \mathrm{~mL}$ volumetric flask, completed with diluent. This solution was further diluted into $25 \mathrm{~mL}$ volumetric flask, dosing $50 \mu \mathrm{L}$. The final concentration (\% level) of the specified impurity was $16.5-18.3 \mathrm{ng} / \mathrm{mL}$ $(0.002 \%)$. The limit of detection values obtained for each impurity and Cabazitaxel are within the acceptance criteria. Detection limit evaluation of CBZ01, CBZ02 and CBZ09 were mentioned in the Table 1.7-1.9.

\section{Limit of quantitation \\ Quantitation limit of CBZ}

The stock solutions of individual specified impurities at concentration level $1.0 \mathrm{mg} / \mathrm{mL}$ were prepared( about $10.000 \mathrm{mg}$ of specified impurity

Table. 2.0: Evaluation of CBZ quantitation limit

\begin{tabular}{|c|c|c|c|c|c|c|c|c|}
\hline S.No & $\begin{array}{c}\text { Sample } \\
\text { weight } \\
(\mathrm{mg})\end{array}$ & $\begin{array}{c}\text { Make up } \\
\text { Volume } \\
(\mathrm{mL})\end{array}$ & $\begin{array}{l}\text { Dilution } \\
(\mu \mathrm{L})\end{array}$ & $\begin{array}{c}\text { Make up } \\
\text { Volume } \\
(\mathrm{mL})\end{array}$ & $\begin{array}{l}\text { Dilution } \\
(\mu \mathrm{L})\end{array}$ & $\begin{array}{c}\text { Make up } \\
\text { Volume } \\
(\mathrm{mL})\end{array}$ & Concentration (\%) & $s / n$ \\
\hline 1 & 25.029 & 25 & 250 & 25 & 200 & 25 & 0.008 & 13.2 \\
\hline 2 & 25.029 & 25 & 250 & 25 & 200 & 25 & 0.008 & 13.2 \\
\hline 3 & 25.029 & 25 & 250 & 25 & 200 & 25 & 0.008 & 13.0 \\
\hline 4 & 25.029 & 25 & 250 & 25 & 200 & 25 & 0.008 & 13.2 \\
\hline 5 & 25.029 & 25 & 250 & 25 & 200 & 25 & 0.008 & 13.4 \\
\hline 6 & 25.029 & 25 & 250 & 25 & 200 & 25 & 0.008 & 13.4 \\
\hline \multicolumn{5}{|c|}{ Mean } & 13.2 & & & \\
\hline
\end{tabular}

standard was weighed into a $10 \mathrm{~mL}$ volumetric flask, dissolved in diluent and filled up with diluent to the volumetric mark. The stock solution was diluted 100 times by dispensing $250 \mu \mathrm{L}$ into a $25 \mathrm{~mL}$ volumetric flask, completed with diluent. This solution was further diluted into $25 \mathrm{~mL}$ volumetric flask, dosing $50 \mu \mathrm{L}$. The final concentration (\% level) of the specified impurity was $16.5-18.3 \mathrm{ng} / \mathrm{mL}$
(0.002\%). Specified impurities mixed solution of the proper concentration was analysed three times. Results of CBZ quantitation limit was mentioned in the Table 2.0.

\section{Quantitation limits of the specified impurities}

Quantitation limit of the specified impurities (CBZM01, CBZN09 and CBZM02) was estimated

Table 2.1: Evaluation of CBZM01 quantitation limit

\begin{tabular}{|c|c|c|c|c|c|c|c|c|}
\hline S.No & $\begin{array}{c}\text { Sample } \\
\text { weight } \\
\text { (mg) }\end{array}$ & $\begin{array}{l}\text { Make up } \\
\text { Volume } \\
\text { (mL) }\end{array}$ & $\begin{array}{l}\text { Dilution } \\
(\mu \mathrm{L})\end{array}$ & $\begin{array}{c}\text { Make up } \\
\text { Volume }(\mathrm{mL})\end{array}$ & $\begin{array}{l}\text { Dilution } \\
(\mu L)\end{array}$ & $\begin{array}{c}\text { Make up } \\
\text { Volume } \\
\text { (mL) }\end{array}$ & Concentration (\%) & $s / n$ \\
\hline 1 & 9.301 & 10 & 250 & 25 & 200 & 25 & 0.008 & 14.8 \\
\hline 2 & 9.301 & 10 & 250 & 25 & 200 & 25 & 0.008 & 14.8 \\
\hline 3 & 9.301 & 10 & 250 & 25 & 200 & 25 & 0.008 & 14.1 \\
\hline 4 & 9.301 & 10 & 250 & 25 & 200 & 25 & 0.008 & 14.5 \\
\hline 5 & 9.301 & 10 & 250 & 25 & 200 & 25 & 0.008 & 14.5 \\
\hline \multirow[t]{2}{*}{6} & 9.301 & 10 & 250 & 25 & 200 & 25 & 0.008 & 14.5 \\
\hline & & Mean & & & & & & 14.5 \\
\hline
\end{tabular}


.Table 2.2: Evaluation of CBZM02 quantitation limit

\begin{tabular}{lcccccccc}
\hline S.No & $\begin{array}{c}\text { Sample } \\
\text { weight } \\
(\mathrm{mg})\end{array}$ & $\begin{array}{c}\text { Make up } \\
\text { Volume } \\
(\mathrm{mL})\end{array}$ & $\begin{array}{c}\text { Dilution } \\
(\mu \mathrm{L})\end{array}$ & $\begin{array}{c}\text { Make up } \\
\text { Volume } \\
(\mathrm{mL})\end{array}$ & $\begin{array}{c}\text { Dilution } \\
(\mu \mathrm{L})\end{array}$ & $\begin{array}{c}\text { Make up } \\
\text { Volume } \\
(\mathrm{mL})\end{array}$ & Concentration $(\%)$ & $\mathrm{s} / \mathrm{n}$ \\
\hline 1 & 9.370 & 10 & 250 & 25 & 200 & 25 & 0.008 & 13.0 \\
2 & 9.370 & 10 & 250 & 25 & 200 & 25 & 0.008 & 13.4 \\
3 & 9.370 & 10 & 250 & 25 & 200 & 25 & 0.008 & 13.4 \\
4 & 9.370 & 10 & 250 & 25 & 200 & 25 & 0.008 & 13.4 \\
5 & 9.370 & 10 & 250 & 25 & 200 & 25 & 0.008 & 14.1 \\
6 & 9.370 & 10 & 250 & 25 & 200 & 25 & 0.008 & 13.0 \\
& \multicolumn{7}{c}{ Mean } \\
\hline
\end{tabular}

Table 2.3: Evaluation of CBZN09 quantitation limit

\begin{tabular}{lcccccccr}
\hline S.No & $\begin{array}{c}\text { Sample } \\
\text { weight } \\
(\mathrm{mg})\end{array}$ & $\begin{array}{c}\text { Make up } \\
\text { Volume } \\
(\mathrm{mL})\end{array}$ & $\begin{array}{c}\text { Dilution } \\
(\mu \mathrm{L})\end{array}$ & $\begin{array}{c}\text { Make up } \\
\text { Volume } \\
(\mathrm{mL})\end{array}$ & $\begin{array}{c}\text { Dilution } \\
(\mu \mathrm{L})\end{array}$ & $\begin{array}{c}\text { Make up } \\
\text { Volume } \\
(\mathrm{mL})\end{array}$ & Concentration $(\%)$ & $\mathrm{s} / \mathrm{n}$ \\
\hline 1 & 9.399 & 10 & 250 & 25 & 190 & 25 & 0.007 & 13.0 \\
2 & 9.399 & 10 & 250 & 25 & 190 & 25 & 0.007 & 13.2 \\
3 & 9.399 & 10 & 250 & 25 & 190 & 25 & 0.007 & 13.2 \\
4 & 9.399 & 10 & 250 & 25 & 190 & 25 & 0.007 & 13.0 \\
5 & 9.399 & 10 & 250 & 25 & 190 & 25 & 0.007 & 13.6 \\
6 & 9.399 & 10 & 250 & 25 & 190 & 25 & 0.007 & 13.9 \\
& \multicolumn{7}{c}{ Mean } \\
\hline
\end{tabular}

based on the detection limit results. Quantitation limit was verified by six injections of each specified impurity solution on the estimated concentration which would result in required $\mathrm{S} / \mathrm{N}<8-15>$. The limit of quantitation values obtained for each impurity and Cabazitaxel are within the acceptance criteria. Determined quantitation limit for CBZM01 is $73 \mathrm{ng} /$ $\mathrm{mL}(0.008 \%)$, for CBZM02 is $71 \mathrm{ng} / \mathrm{mL}(0.008 \%)$ and for CBZN09 is $66 \mathrm{ng} / \mathrm{mL}(0.007 \%)$. The detailed results regarding quantitation limit of various impurities were listed in the Table 2.1-2.3.

\section{Linearty and Range}

The linearity is determined by injecting the solutions in duplicate containing known impurities and Cabazitaxel ranging from 0.05 to $1.13 \%$ and impurities ranging from $0.05 \%$ to $0.22 \%$ of the specified limit. Regression analysis was performed and correlation coefficient was determined (Table 2.4 and Figure 2.0). Response factor for each impurity was determined with respect to Cabazitaxel.

The linearity range as the range for determining the impurities were reported. The details of the results were presented in the Table 2.5-2.7 and Figure 2.1-2.3 show the line of best fit for peak area versus concentration for each impurity. The linearity results for Cabazitaxel and all the impurities in the specified concentration range are found satisfactory, with a correlation coefficient greater than 0.99 . 
Table 2.4: Linearity of Cabazitaxel

\begin{tabular}{lcc}
\hline $\begin{array}{l}\text { \% Level of } \\
\text { Cabazitaxel }\end{array}$ & $\begin{array}{c}\text { Concentration } \\
(\mu \mathrm{g} / \mathrm{mL})\end{array}$ & $\begin{array}{c}\text { Average } \\
\text { peak } \\
\text { area }\end{array}$ \\
\hline 30 & 0.280 & 1468 \\
60 & 0.555 & 2530 \\
80 & 0.742 & 3342 \\
100 & 0.925 & 4063 \\
120 & 1.119 & 4944 \\
150 & 1.391 & 6023 \\
220 & 2.052 & 8775 \\
Slope & 5514 & \\
Intercept & 166.7 & \\
Correlation coefficient $\left(\mathrm{R}^{2}\right)$ & 0.9998 & \\
\hline
\end{tabular}

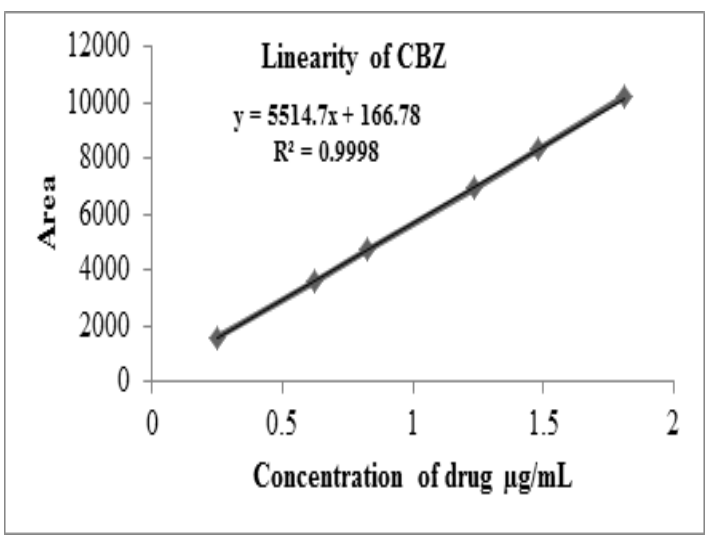

Fig. 2.0: Linearity graph of Cabazitaxel

Table 2.5: Linearty of CBZM01

\begin{tabular}{lcc}
\hline $\begin{array}{l}\text { \% Level } \\
\text { CBZM01 }\end{array}$ & $\begin{array}{c}\text { Concentration } \\
(\mu \mathrm{g} / \mathrm{mL})\end{array}$ & $\begin{array}{c}\text { Average } \\
\text { peak area }\end{array}$ \\
\hline 20 & 0.274 & 1148 \\
50 & 0.696 & 3019 \\
70 & 0.912 & 4020 \\
100 & 1.373 & 5886 \\
120 & 1.646 & 7094 \\
150 & 2.005 & 8661 \\
\hline
\end{tabular}

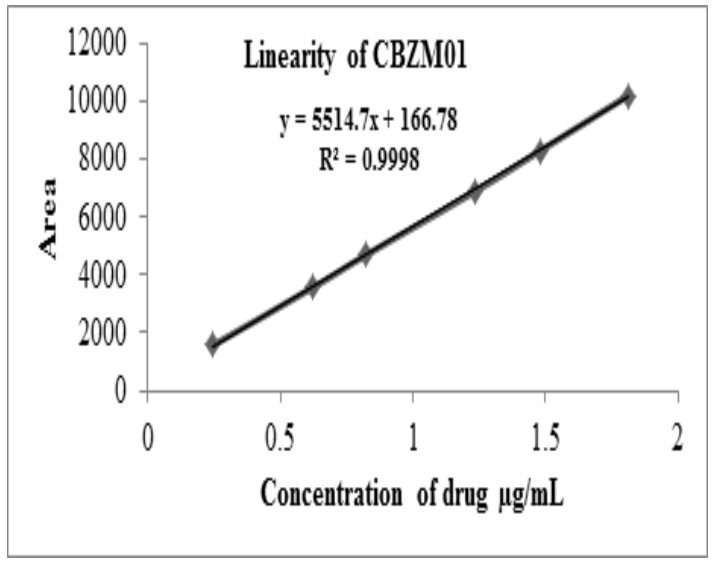

Fig. 2.1: Linearity graph of CBZM01 Table 2.6: Linearity of CBZM02

\begin{tabular}{lcc}
\hline $\begin{array}{l}\text { \% Level } \\
\text { of CBZM02 }\end{array}$ & $\begin{array}{c}\text { Concentration } \\
(\mu \mathrm{g} / \mathrm{mL})\end{array}$ & $\begin{array}{c}\text { Average } \\
\text { peak area }\end{array}$ \\
\hline 20 & 0.268 & 1081 \\
50 & 0.681 & 2715 \\
70 & 0.893 & 3527 \\
100 & 1.345 & 5390 \\
120 & 1.607 & 6290 \\
150 & 1.964 & 7690 \\
\hline
\end{tabular}

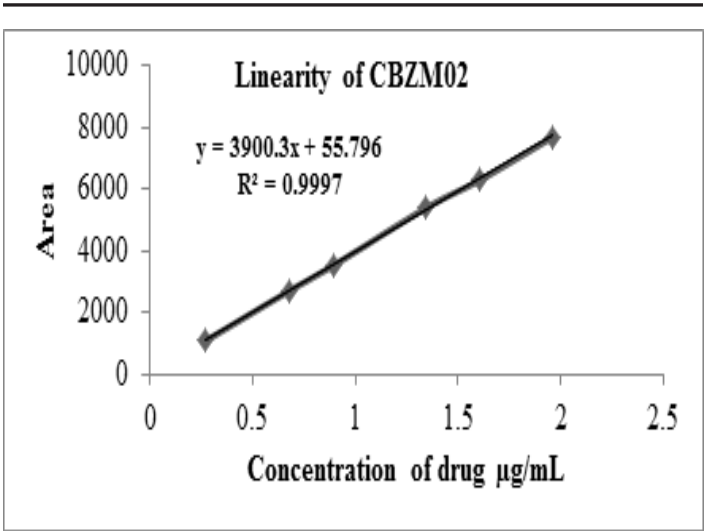

Fig. 2.2: Linearity graph of CBZMO2 Table 2.7: Linearity of CBZN09

\begin{tabular}{lcc}
\hline $\begin{array}{l}\% \text { Level } \\
\text { of CBZN09 }\end{array}$ & $\begin{array}{c}\text { Concentration } \\
(\mu \mathrm{g} / \mathrm{mL})\end{array}$ & $\begin{array}{c}\text { Average } \\
\text { peak area }\end{array}$ \\
\hline 20 & 0.247 & 1578 \\
50 & 0.625 & 3592 \\
70 & 0.823 & 4698 \\
100 & 1.234 & 6905 \\
120 & 1.480 & 8312 \\
150 & 1.811 & 10217 \\
\hline
\end{tabular}




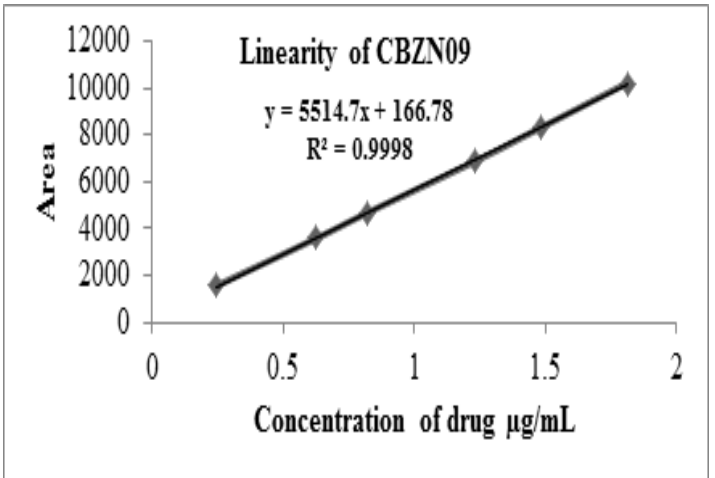

Fig. 2.3: Linearity graph of CBZN09

\section{Accuracy}

Analyses performed triple injections performed on $0.10 \%, 0.15 \%$ and $0.22 \%$ of the nominal concentration, respectively) were used. From linear regressions, theoretical contents corresponding to the peak areas obtained from the triple analyses and deviations of these values from the actual concentrations were calculated. The percentage recovery values obtained for each impurity are in the range of about $97.3-101.8$, which are within the specified criteria. Summary of \% recovery of $\mathrm{CBZ}$ and its impurities were listed in the Table 2.8-3.1.

Table 2.8: Summary of \% recoveries for Cabazitaxel

\begin{tabular}{lcccc} 
\% of Cabazitaxel & Theoretical conc. $(\mathrm{mg} / \mathrm{mL})$ & Measured conc. $(\mathrm{mg} / \mathrm{mL})$ & \% Recovery & Average \\
\hline \multirow{2}{*}{ LOQ } & 0.2798 & 0.2677 & 95.7 & 96.8 \\
& 0.2798 & 0.2692 & 96.2 & \\
& 0.2798 & 0.2641 & 94.4 & \\
$100 \%$ & 0.9249 & 0.9157 & 99.0 & 99.1 \\
& 0.9249 & 0.9184 & 99.3 & \\
& 0.9249 & 0.9160 & 99.0 & \\
$150 \%$ & 1.3907 & 1.3889 & 99.9 & 99.9 \\
& 1.3907 & 1.3848 & 99.6 & \\
$220 \%$ & 1.3907 & 1.3957 & 100.4 & 100 \\
& 2.0520 & 2.0534 & 100.1 & \\
& 2.0520 & 2.0495 & 99.9 & \\
& 2.0520 & 2.0509 & 100.0 & \\
\hline
\end{tabular}

Table 2.9: Summary of \% recoveries for CBZM01

\begin{tabular}{lcccc}
\hline \% of CBZM01 & Theoretical conc. $(\mathrm{mg} / \mathrm{mL})$ & Measured conc. $(\mathrm{mg} / \mathrm{mL})$ & \% Recovery & Average \\
\hline \multirow{2}{*}{ LOQ } & & & & \\
& 0.2743 & 0.2728 & 99.5 & 98.8 \\
& 0.2743 & 0.2733 & 99.6 & \\
$100 \%$ & 0.2743 & 0.2712 & 98.9 & \\
& 1.3735 & 1.3630 & 99.2 & 99.1 \\
& 1.3735 & 1.3634 & 99.3 & \\
$150 \%$ & 1.3735 & 1.3588 & 98.9 & \\
& 2.0054 & 2.0062 & 100.0 & 100.1 \\
& 2.0054 & 2.0104 & 100.3 & \\
& 2.0054 & 2.0027 & 99.9 & \\
\hline
\end{tabular}


Table 3.0: Summary of $\%$ recoveries for CBZM02

\begin{tabular}{lcccc}
\hline \% of CBZM02 & $\begin{array}{c}\text { Theoretical conc. } \\
(\mathrm{mg} / \mathrm{mL})\end{array}$ & $\begin{array}{c}\text { Measured conc. } \\
(\mathrm{mg} / \mathrm{mL})\end{array}$ & \% Recovery & Average \\
\hline \multirow{2}{*}{ LOQ } & 0.2679 & 0.2606 & 97.3 & 97.3 \\
& 0.2679 & 0.2608 & 97.4 & \\
$100 \%$ & 0.2679 & 0.2639 & 98.5 & 101.8 \\
& 1.3447 & 1.3678 & 101.7 & \\
& 1.3447 & 1.3678 & 101.7 & 99.3 \\
$150 \%$ & 1.3447 & 1.3691 & 101.8 & \\
& 1.9643 & 1.9575 & 99.7 & \\
& 1.9643 & 1.9455 & 99.0 & \\
& 1.9643 & 1.9467 & 99.1 & \\
\hline
\end{tabular}

Table 3.1: Summary of $\%$ recoveries for CBZN09

\begin{tabular}{|c|c|c|c|c|}
\hline$\%$ of CBZN09 & $\begin{array}{l}\text { Theoretical conc. } \\
(\mathrm{mg} / \mathrm{mL})\end{array}$ & $\begin{array}{l}\text { Measured conc. } \\
(\mathrm{mg} / \mathrm{mL})\end{array}$ & $\%$ Recovery & Average \\
\hline \multirow[t]{3}{*}{ LOQ } & 0.2467 & 0.2364 & 95.8 & 97.1 \\
\hline & 0.2467 & 0.2352 & 95.3 & \\
\hline & 0.2467 & 0.2359 & 95.6 & \\
\hline \multirow[t]{3}{*}{$100 \%$} & 1.2344 & 1.2219 & 99.0 & 98.1 \\
\hline & 1.2344 & 1.2063 & 97.7 & \\
\hline & 1.2344 & 1.2031 & 97.5 & \\
\hline \multirow[t]{3}{*}{$150 \%$} & 1.8107 & 1.8225 & 100.7 & 100.4 \\
\hline & 1.8107 & 1.8120 & 100.1 & \\
\hline & 1.8107 & 1.8187 & 100.4 & \\
\hline
\end{tabular}

\section{RESULTS AND DISCUSSION}

A simple, economic, accurate and precise reverse phase liquid chromatography method was successfully developed using Zorbax SB C18 (100 $\mathrm{mm} \times 3.0 \mathrm{~mm}, 1.8 \mu \mathrm{m}$ particle size). Injection volume of $3 \mu \mathrm{L}$ is injected and eluted with the mobile phase eluent-A: pH 3.0 phosphate buffer and eluent-B: acetonitrile, which is pumped at a flow rate of 0.8 $\mathrm{mL} / \mathrm{min}$. Detection, was carried out at $220 \mathrm{~nm}$.

For Selectivity, the chromatograms were recorded for standard and sample solutions of Cabazitaxel and its related substances. Selectivity studies reveal that the peak is well separated from each other. Therefore the method is selective for the determination of related substances in Cabazitaxel.

The limit of detection (LOD) and limit of quantitation (LOQ) for CBZM01 impurity $0.002 \%$ and $0.008 \%$, CBZM02 impurity $0.002 \%$ and $0.008 \%$, CBZN09 impurity $0.002 \%$ and $0.007 \%$ and Cabazitaxel $0.002 \%$ and $0.008 \%$ respectively. Using the optimized chromatographic conditions, the retention times (in min) of impurities were 6.297 for CBZM01 impurity, 17.417 for CBZM02, 10.669 for CBZN09 and 11.021 for Cabazitaxel. The linearity results for $C$ abazitaxel and all the impurities 
in the specified concentration range are found satisfactory, with a correlation coefficient greater than 0.99.Calibration curve was plotted and correlation coefficient for Cabazitaxel and its impurities found to be $0.9997,0.9997,0.9998$ and 0.9998 respectively.

The accuracy studies were shown as \% recovery for Cabazitaxel and its impurities at specification level. The limit of \% recovered shown is in the range of 90 and $110 \%$ and the results obtained were found to be within the limits. Hence the method was found to be accurate. The accuracy studies showed \% recovery of the Cabazitaxel and its related substances in the range 97.3 to101.8 respectively.
For Precision studies six replicate injections were performed. \%RSD was determined from the peak areas of Cabazitaxel and its impurities. The acceptance limit should be not more than 10 , and the results were found to be within the acceptance limits.

\section{CONCLUSIONS}

Chromatographic method developed by the author for the determination of Cabazitaxel and its related substances was rapid, simple, sensitive, precise, and accurate. Therefore, the proposed method can be successfully applied for the routine analysis of the active pharmaceutical ingredients for assurance of its quality during its formulation.

\section{REFERENCES}

1. Cheetham, P.; Petrylak,D.P. Tubulin-targeted agents including docetaxel and Cabazitaxel. Cancer Jour. 2013, 19(1), 59-65.

2. Assessment Report for Jevtana (Cabazitaxel), Procedure No: EMEA/ H/C/ 002018, European Medicines Agency, London, 2011.

3. Jevtana (Cabazitaxel) Injection approved by U.S. FDA after priority review" (Press release). Sanofi-Aventis, 2010.

4. Jordan, M.A.; Wilson, L. Microtubules as targets for anticancer drugs. Nat. Rev. Cancer. 2004, 4(4), 253-65.

5. Pivot,X.; Koralewski, P.; Hidalgo, J.L.; Chan,A.; Goncalves, A.; Schwartsmann, G.; Assadourian, S.; Lotz, J.P. A multicenter phase II study of XRP6258 administered as a 1-h I.V. infusion every 3 weeks in taxane-resistant metastatic breast cancer patients. Annals of Oncology. 2008, 19(9), 1547-1552.

6. Kishore, G. New spectrophotometric methods for the quantitative estimation of Cabazitaxel in formulations. Int. J.of Res. and Reviews in Pharmacy and Appl. sci. 2012, 2(5), 950-958.
7. Mathrusri Annapurna, M.; Pramadvara, K.; Venkatesh, B.; Sowjanya, G. Stabilityindicating RP-HPLC method for the determination of Cabazitaxel. Indo Amer. J. of Pharm. Res. 2013, 3(11), 9262-9269.

8. Chengyan, Li.; Gongjian,Lan.; Jinyuan Jiang.; Mingjie Sun.; Taijun Hang. Development and validation of a stabilityindicating HPLC method for the determination of the impurities in Cabazitaxel. Chromatographia. 2015, 78, 825-831.

9. Mathrusri Annapurna, M.; Venkatesh, B.; Naga Supriya, G. A validated stabilityindicating liquid chromatographic method for determination of Cabazitaxel-A novel microtubule inhibitor. J. of Bioequivalence and Bioavailability. 2014, 6(4), 134-138.

10. Mathrusri Annapurna, M.; Venkatesh, B.; Pramadvara, K.; Hemchand, S. Development and validation of a stabilityindicating liquid chromatographic method for the assay of Cabazitaxel. Chemical science transactions. 2014, 3(2), 854-60. 
11. Jagannath Patrol, V.; Nageshwara Rao, R.; Tripathy, N.K. LC-MS/MS determination of Cabazitaxel in rat whole blood on dry blood Spots. Open Access Scientific Reports. 2012, 1 (6), 1-4.

12. Kort, A.; Hillebrand, M.J.X.; Cirkel, G.A.; Voest, E.E.; Schinkel,A.H.; Rosing, H.; Schellen, J.H.M.; Beijnen, J.H. Quantification of Cabazitaxel, its metabolite docetaxel and the determination of the demethylated metabolites RPR112698 and RPR123142 as docetaxel equivalents in human plasma by liquid chromatography tandem mass spectrometry. J. of Chromatography B. 2013, 925,117-123.

13. Peter de, Bruijn.; Anne-Joy, M. de Graan.; Annemieke, Nieuweboer.; Ron, H.J. Mathijssen.; Mei-Ho, Lam.; Ronald de ,Wit.; Erik, A.C. Wiemer.; Walter, J.Loos. Quantification of Cabazitaxel in human plasma by liquid chromatography/ triplequadrupole mass spectrometry: A practical solution for non-specific binding. J. of Pharm and Biomed. Anal. 2012, 59,117-22. 